\title{
Identificación de nematodos gastrointestinales en búfalos faenados en un frigorífico de Corrientes, Argentina
}

\author{
Lobayan, S.I. ${ }^{1}$; Schapiro, J.H.'; Fiel, C.A. ${ }^{3}$; Ostriñuk, R. ${ }^{4}$; Genessini, R.M. ${ }^{4}$ \\ ${ }^{1}$ Escuela Veterinaria, Univ.Salvador (USAL), Virasoro (Corrientes, Argentina); ${ }^{2}$ Escuela Veterinaria, \\ USAL (Pilar); ${ }^{3}$ Univ.Nac.Centro Prov.Bs.As.; ${ }^{4}$ Alumnos practicantes USAL. \\ E-mail: sergio.lobayan@usal.edu.ar
}

\begin{abstract}
Resumen
Lobayan, S.I.; Schapiro, J.H.; Fiel, C.A.; Ostriñuk, R.; Genessini, R.M.: Identificación de nematodos gastrointestinales en búfalos faenados en un frigorífico de Corrientes, $\mathrm{Ar}$ gentina. Rev. Vet. 31: 1, 95-96, 2020. El objetivo del trabajo fue identificar y cuantificar los ejemplares adultos de nematodos gastrointestinales en los búfalos faenados en el frigorífico de Virasoro (Corrientes) mediante la necropsia parasitaria del tubo digestivo y, por intermedio de estudios coprológicos, correlacionarlos con el recuento de huevos por gramo de materia fecal y la proporción de géneros parasitarios de larvas de tercer estadio de los coprocultivos. Se realizaron en total 4 necropsias parasitarias que correspondieron a búfalos machos jóvenes, de los cuales el 50\% presentó ejemplares adultos de Trichostrongylus sp y Haemonchus $s p$ ubicados solamente en el abomaso. En los estudios coprológicos, el $75 \%$ de las muestras presentaron recuentos de huevos por gramo de materia fecal, de los cuales únicamente en dos casos se pudieron identificar larvas de tercer estadio de Haemonchus $s p$ en los coprocultivos.
\end{abstract}

Palabras clave: búfalos, nematodos gastrointestinales, larvas, necropsia parasitaria.

\begin{abstract}
Lobayan, S.I.; Schapiro, J.H.; Fiel, C.A.; Ostriñuk, R.; Genessini, R.M.: Identification of gastrointestinal nematodes in buffaloes slaughtered in Corrientes, Argentina. Rev. Vet. 31: 1, 95-96, 2020. The objective of this work was to identify and quantify the adult specimens of gastrointestinal nematodes in buffaloes slaughtered in Virasoro (Corrientes, Argentina) by means of necropsy of the digestive tract, and through coprological studies, in order to correlate them with the count of eggs per gram of fecal stool and the proportion of parasite genera of third stage larvae of stool culture. A total of 4 necropsies corresponding to young male buffaloes were carried out, of which $50 \%$ presented adult specimens of Trichostrongylus $s p$ and Haemonchus sp located only in the abomasum. In the coprological studies, $75 \%$ of the samples presented counts of egg per gram of fecal stool, with only 2 cases with the identification of third stage larvae of Haemonchus sp in stool cultures.
\end{abstract}

Key words: buffaloes, gastrointestinal nematodes, larvae, parasitical necropsy.

\section{INTRODUCCIÓN}

El objetivo de este trabajo fue identificar y cuantificar los ejemplares adultos de nematodos gastrointestinales en búfalos faenados en el frigorífico de Gobernador Virasoro (Corrientes).

Se efectuaron necropsias parasitarias del tubo digestivo y, por intermedio de estudios coprológicos, se correlacionaron con el recuento de huevos por gramo de materia fecal y con la proporción de géneros parasitarios de larvas de tercer estadio de coprocultivos.

\section{MATERIAL Y MÉTODOS}

Se utilizó un diseño de investigación observacional transversal. La selección de los animales se efectuó por

Recibido: agosto 2019 / Aceptado: noviembre 2019 muestreo de conveniencia y se realizó de acuerdo a la disponibilidad y disposición de la lista de matanza del frigorífico.

En la necropsia se identificaron y cuantificaron los ejemplares adultos de nematodes gastrointestinales del tubo digestivo de cada búfalo, mediante la técnica propuesta por el INTA ${ }^{2}$, siguiendo los lineamientos de la World Association for the Advancement of Veterinary Parasitology ${ }^{6}$.

Para realizar los estudios coprológicos, previamente a la faena se extrajo materia fecal del recto de cada búfalo, ejecutando luego el recuento de huevos por gramo (HPG) mediante la técnica de McMaster modificada ${ }^{4}$. Con las muestras obtenidas se efectuaron los coprocultivos utilizando la técnica de Corticelli ${ }^{1}$ y se identificaron y cuantificaron los géneros parasitarios de larvas de tercer estadio siguiendo el método y las claves propuestas por $\mathrm{Niec}^{3}$. 
Los resultados de la necropsia parasitaria se presentaron en relación a la presencia-ausencia de nematodes gastrointestinales y cantidad de géneros parasitarios por órganos, y los resultados del estudio se expusieron en HPG y porcentual de géneros de nematodes en el coprocultivo.

\section{RESULTADOS}

Tabla 1. Resultados de la necropsia parasitaria (NP) y los estudios coprológicos (EC).

\begin{tabular}{ccccc}
\hline & \multicolumn{2}{c}{ NP } & \multicolumn{2}{c}{ EC } \\
\hline $\mathrm{Bu}$ & $T . s p$ & H. $s p$ & HPG & H. $s p$ \\
\hline 1 & 0 & 0 & 10 & 0 \\
2 & 100 & 100 & 10 & $100 \%$ \\
3 & 0 & 400 & 60 & $100 \%$ \\
4 & 0 & 0 & 0 & 0 \\
\hline
\end{tabular}

NP: necropsia parasitaria; EC: estudio coprológico, T.sp.: Trichostrongilus sp, H.sp:Haemonchus sp, Bu: bubillos, HPG: huevos por gramo de materia fecal.

Se realizaron en total 4 necropsias que correspondieron a búfalos machos jóvenes (bubillos), de los cuales el $50 \%$ presentaron ejemplares adultos de Trichostrongylus sp. y Haemonchus sp. ubicados solamente en el abomaso. En el estudio coprológico el $75 \%$ de las muestras presentaron recuentos de HPG, de los cuales únicamente en dos ejemplares se pudieron identificar larvas de tercer estadio de Haemonchus sp. en los coprocultivos. Los resultados se observan en la Tabla 1.

\section{CONCLUSIONES}

En este estudio los nematodes gastrointestinales se identificaron a nivel de género, no pudiendo determinarse a qué especie pertenecían. Teniendo en cuenta que en la región mesopotámica, muchos de los sistemas productivos son extensivos-mixtos, con rodeos bovinos-bubalinos-ovinos, las especies de nematodes y en especial Haemonchus sp, podrían encontrarse en otros hospedadores de los rodeos, por lo cual un hospe- dador podría estar co-infectado por más de una especie de Haemonchus sp.

En algunos casos la presencia de ejemplares adultos en el tubo digestivo no se correlacionó con los resultados del HPG y los coprocultivos, lo cual podría explicarse, como sucede en los bovinos, por la edad de los hospedadores, ya que la susceptibilidad de los animales a los parásitos está relacionada con el desarrollo de inmunidad, la cual depende del tiempo de exposición y de la carga de parásitos ${ }^{5}$. El tamaño de la muestra fue insuficiente como para permitir determinar alguna correlación entre los hallazgos de la necropsia parasitaria y los estudios coprológicos.

Esta comunicación evidencia la necesidad de profundizar los estudios epidemiológicos sobre las parasitosis de la región, a efectos de establecer medidas de control que reduzcan su impacto en la ganadería bubalina.

\section{REFERENCIAS}

1. Corticelli B, Lai M. 1963. Studies on the technique of culture of infective larvae of gastrointestinal strongyles of cattle. Acta Med Vet Napoli 9: 347-357.

2. Lukovich R. 1981. Identificación de las formas adultas de los nematodes gastrointestinales y pulmonares de los rumiantes en la República Argentina. Boletín Técnico del INTA. http://cnia.inta.gov.ar/helminto.

3. Niec R. 1968. Cultivo e identificación de larvas infectantes de nematodes gastrointestinales del bovino y ovino. Manual Técnico INTA 3: 1-37.

4. Roberts F, O'Sullivan P. 1949. Methods for egg count and larval cultures for strongyles infesting gastrointestinal tract of cattle. Aust J Agric Res 1: 99-102.

5. Steffan PE et al. 1994. Efectos en producción y control de nematodes gastrointestinales en bovinos. En: Nari, A.y Fiel, $C$. A. (Eds.), Enfermedades parasitarias de importancia económica en bovinos.. Edit.Hemisferio Sur, p. 131153.

6. Wood IB et al. 1995. Guidelines for evaluating the efficacy of anthelmintics in ruminants (bovine and ovine). Vet Parasitology 10: 205-264. 